\title{
Les mécanismes de contrôle de la méiose dans la lignée germinale mâle
}

\author{
P. DURAND
}

INSERM-INRA U 418, Hôpital Debrousse, 69322 Lyon Cedex 05

\section{RESUME}

En 1883 il fut observé que, tandis que l'œuf fécondé d'un ver (Parascaris Equorum) possédait quatre chromosomes, le noyau de l'ovocyte, comme celui du gamète mâle, contenait seulement deux chromosomes. Cette découverte impliquait que les cellules germinales devaient être formées lors d'un processus de division cellulaire particulier, la méiose, conduisant à une division par deux du nombre de chromosomes présents dans les cellules somatiques de l'individu. La mitose et la méiose partagent un grand nombre d'éléments régulateurs. Parmi ceux-ci, les kinases dépendantes des cyclines (CDK) et les cyclines apparaissent les plus importantes car ces activités sont responsables de la progression des cellules dans le cycle cellulaire. L'activité des CDK (actuellement au nombre de 7) est régulée par leur association avec des cyclines spécifiques ( 8 types de cyclines ont actuellement été identifiés), ainsi que par des réactions de phosphorylation/déphosphorylation et par l'association à des petites molécules capables de lier les complexes CDK-cyclines et d'inhiber leur activité kinase : les CDKI. Les protéines Rb et p53 apparaissent également fondamentales pour la régulation de ces processus. La plupart des travaux réalisés sur le déroulement de la méiose chez les eucaryotes supérieurs l'ont été en utilisant l'ovocyte de mollusques et d'amphibiens, et plus rarement, de mammifères. Ils ont permis de mettre en évidence les activités MPF (pour maturation promoting factor) $=$ p34 cdc2-cycline B' et CSF (pour cytostatic factor) = très vraisemblablement le couple MOS/MAP kinase, qui apparaissent responsables des phénomènes de reprise de la méiose en prophase I et de blocage en métaphase II. Les facteurs de signalisation cellulaire impliqués dans la régulation de ces phénomènes incluent l'AMPc, le Ca++ et les agonistes de la PKC. Beaucoup moins de données sont actuellement disponibles pour ce qui est du contrôle de la méiose dans la lignée spermatogénétique. Ceci tient, en particulier, à la difficulté d'obtenir des cellules germinales à un stade précis de leur différenciation en grande quantité, et au manque de systèmes in vitro permettant d'appréhender l'importance des régulations post-traductionnelles impliquées dans ces processus. Il ressort toutefois des travaux ayant pour but de rechercher l'expression des gènes codant pour les protéines régulant le cycle cellulaire que la majorité de ceux-ci sont exprimés dans le testicule (c-mos, cyclines, CDK, cdc25 ...) d'une manière dépendante du type celIulaire et du stade de développement. Les rares études réalisées sur le 
contrôle de la méiose par les hormones et les facteurs locaux de régulation indiquent que la FSH, l'interleukine-1 $\alpha$ et le MIP-1a stimulent l'incorporation de thymidine tritiée par les spermatocytes préleptotènes, contrairement à l'interleukine-6 qui l'inhibe. Le développement récent, dans notre laboratoire, de deux systèmes de coculture cellules somatiques/cellules germinales testiculaires permettant le maintien d'une activité transcriptionnelle des cellules germinales pendant deux à trois semaines devrait permettre de reprendre ces études et de hiérarchiser l'importance des facteurs locaux impliqués dans le déroulement des étapes mitotique et méiotique de la spermatogenèse.

Mots-clés : Méiose, cycle cellulaire, ovocyte, spermatogenèse.

\section{INTRODUCTION}

La découverte que les cellules germinales sont haploïdes et, en conséquence, doivent résulter d'un type particulier de division cellulaire, provient d'une observation qui fut parmi les premières à suggérer que ce sont les chromosomes qui portent l'information génétique. En 1883 il fut observé que, tandis que l'œuf fécondé d'un ver (Parascaris Equorum) possédait quatre chromosomes, le noyau de l'ovocyte, comme celui du gamète mâle, contenait seulement deux chromosomes. Cela conduisait à pouvoir expliquer que malgré la différence de taille très importante entre les gamètes mâle et femelle la contribution paternelle et maternelle aux caractéristiques du descendant apparaissait souvent d'importance égale. Cette découverte impliquait également que les cellules germinales devaient être formées lors d'un processus particulier de division cellulaire conduisant à une division par deux du nombre de chromosomes présents dans les cellules somatiques de l'indi- vidu. C'est ce type de division cellulaire particulier qui est appelé méiose. Ce terme provient d'une racine grecque signifiant "diminution". Le comportement des chromosomes lors de la méiose est bien plus complexe que les premières études le laissaient supposer, et ce n'est que dans les années 1930 que les particularités essentielles de la méiose furent décryptées.

\section{LA MEIOSE IMPLIQUE DEUX DIVISIONS NUCLEAIRES AU LIEU D'UNE SEULE}

A l'exception des chromosomes sexuels, un noyau diploïde contient deux représentants de chacun des autres chromosomes (les autosomes), l'un venant du parent mâle, l'autre venant du parent femelle. Ces deux "versions" du même chromosome sont appelées homologues et, dans la plupart des cellules, chaque homologue a une existence indépendante. Quand chacun des chromosomes est dupliqué par replication de l'ADN, les deux copies du chromosome dupliqué restent dans un premier temps associées, c'est ce qu'on appelle les chromatides soeurs. Dans une division cellulaire ordinaire les deux chromatides soeurs s'alignent sur le fuseau de division puis elles se séparent l'une de l'autre à l'anaphase pour chacune devenir un chromosome individualisé. De cette manière, chacune des cellules filles hérite d'une copie de chaque chromosome paternel et de chaque chromosome maternel.

Au contraire, un gamète haploïde, produit par la division d'une cellule diploïde lors d'une méiose, doit contenir la moitié du nombre original de chromosomes : seulement un chromosome à la place de chaque paire de chromosomes homologues. Ainsi, chaque gamète est formé avec soit la copie paternelle, soit la copie maternelle de chacun des gènes de la cellule. Cette particularité nécessite une machinerie cellulaire de division particulière. Ce mécanisme nécessite que 
chaque homologue reconnaisse son partenaire et lui soit physiquement associé sur le fuseau de division. Cette association entre chromosomes paternels et maternels est particulière à la méiose. Les mécanismes de reconnaissance entre homologues paternels et maternels sont encore très mal connus.

Ainsi, une méiose pourrait-elle se réaliser seulement comme une simple mitose dans laquelle il n'y aurait pas de phase de synthèse d'ADN. En fait le processus est beaucoup plus complexe. Avant que les paires de chromosomes homologues soient formées, chacun se duplique pour produire deux chromatides filles comme dans une mitose ordinaire. C'est seulement après la replication de l'ADN que les particularités de la méiose apparaissent. Plutôt que de se séparer, les deux chromatides filles se comportent comme une seule entité, comme si la duplication du chromosome n'avait pas eu lieu. Chaque homologue dupliqué s'accole à son partenaire pour former un divalent qui contient quatre chromatides. Cette association par paire permet des recombinaisons génétiques, c'est-à-dire qu'un fragment de chromosome maternel, par exemple, peut être échangé avec le fragment correspondant du chromosome paternel. Le bivalent se positionne ensuite sur le fuseau et, à l'anaphase, les deux homologues dupliqués (chacun constitué de deux chromatides soeurs) se séparent et se dirigent vers des pôles opposés. Puisque chacune des chromatides soeurs se comporte comme une seule entité, chaque cellule fille hérite de deux copies de l'un des deux homologues quand la cellule se divise. Ces deux copies sont identiques, à l'exception des recombinaisons génétiques qui peuvent avoir eu lieu. Les deux cellules résultant de cette division (division I de la méiose) contiennent ainsi une quantité d'ADN diploïde mais différent d'une cellule diploïde normale en deux points :

- chacune des deux copies d'ADN de chaque chromosome dérive de seulement un des deux chromosomes homologues de la cellule d'origine (sauf quand il y a eu recombinaison génétique).

- Ces deux copies sont héritées sous forme de chromatides soeurs en étroite association, comme si elles n'étaient qu'un seul chromosome.

La formation des cellules gamétiques s'effectue dès lors, simplement, par une seconde division cellulaire - division II de méiose - sans duplication d'ADN. Les chromosomes s'alignent sur un second fuseau de division et les chromatides soeurs se séparent, comme dans une mitose normale, produisant ainsi les cellules haploïdes. Ainsi la méiose est composée de deux divisions cellulaires suivant une seule phase de synthèse d'ADN, de sorte que quatre cellules haploïdes sont formées à partir de chaque cellule entrant en méiose.

\section{QUELS SONT LES GENES IMPLIQUES DANS CES PROCESSUS ?}

La méiose apparait donc comme un type particulier de division cellulaire opérant dans les cellules germinales. Ces deux types de division cellulaire (mitose et méiose) partagent donc un grand nombre de gènes régulateurs. D'ailleurs, la connaissance que l'on a, à l'heure actuelle, de ces mécanismes, provient en particulier de recherches effectuées sur l'ovocyte d'amphibien et sur la levure, ainsi que sur des cellules somatiques en division synchronisée. Au coeur de ces mécanismes, il y a les CDK, c'est-à-dire les kinases dépendantes des cyclines, dont les activités sont responsables de la progression des cellules dans le cycle cellulaire. Le cycle cellulaire décrit les étapes conduisant à la duplication des cellules somatiques. Il est similaire chez tous les eucaryotes, de la levure à l'homme, et il est composé de 5 phases successives, G0, G1, $\mathrm{S}, \mathrm{G} 2$ et $\mathrm{M}$. Les phases $\mathrm{S}$ et M, temps moteurs du cycle, sont séparées par des intervalles (gap en anglais, d'où le G), les phases $\mathrm{G} 1$ et G2. 
G0 est une phase de quiescence cellulaire durant laquelle l'activité métabolique est faible. Sa durée est variable selon le type cellulaire, de quelques secondes à quelques années. En réponse à un stimulus, la cellule quiescente entre dans le cycle et se divise. Pendant la phase G1, dont la durée varie de 10 heures à quelques jours, la taille de la cellule augmente et la synthèse des protéines nécessaire au passage en phase $S$ s'effectue. Cette phase $\mathbf{S}$ (qui est la phase de synthèse de l'ADN) dure en moyenne 6 heures mais il peut exister des variations relativement importantes selon le type cellulaire. A l'issue de cette phase $\mathrm{S}$ la cellule est devenue tétraploïde. G2, phase de repos apparent, prépare la cellule à la mitose; elle dure en moyenne 4 heures. Enfin, la phase $\mathrm{M}$, ou mitose, correspond à la division de la cellule. Elle dure environ 2 heures et s'achève par la séparation des cellules filles. La progression des cellules dans le cycle est contrôlée par l'activation et l'inactivation des kinases dépendantes des cyclines, les CDK. L'activité des complexes cyclines-CDK est elle-même régulée par des phosphorylations, ainsi que par leur liaison avec des inhibiteurs de cyclines-kinases, les CKI.

\section{Les CDK, les cyclines, les CKI}

C'est en 1988 que le facteur MPF contrôlant la transition G2/M a été identifié. A l'origine MPF signifiait "maturation promoting factor", car c'est un facteur qui permet la reprise de la méiose d'un ovocyte bloqué. Puisque le MPF apparaît contrôler la transition G2/M à la fois lors de la méiose et lors de la mitose, l'acronyme MPF est resté mais la signification de celui-ci a changé pour signifier "M phase promoting factor". Le MPF a été identifié d'un point de vue moléculaire comme étant un complexe kinase hétérodimérique constitué d'une sous-unité catalytique, $\mathrm{p} 34 \mathrm{cdc} 2$, et d'une sous-unité régulatrice, la cycline $B$ et qui présente une forte préférence pour l'histone $\mathrm{H} 1$ comme substrat. Depuis lors, il est apparu que l'ensemble des transitions du cycle cellulaire étaient vraisemblablement contrôlées par de tels complexes entre différents membres d'une famille de sérine/thréonine kinases apparentées à cdc2, les CDK et leurs partenaires cyclines respectifs. Pas moins de sept CDK (CDKI à CDK7, cdc2 étant CDKI) ont été clonées. De taille homogène (33 à 35 $\mathrm{kDa}$ ), elles présentent entre elles des homologies de séquence supérieures à $40 \%$, en particulier au sein d'une région de 300 acides aminés comprenant le site actif de l'enzyme et son site de liaison avec les unités régulatrices que sont les cyclines.

Les cyclines doivent leur nom à leur expression périodique au cours du cycle. Leur concentration intracellulaire oscille au cours du cycle : elles s'accumulent puis disparaissent pour s'accumuler à nouveau et redisparaître. Une cycline est définie par une séquence protéique qui comprend un domaine conservé d'environ 100 acides aminés responsables de la liaison et de l'activité de la CDK. Huit types de cycline ont été clonés chez les mammifêres (A, B, C, D, E, F, G, H). Le rôle et le niveau d'intervention des différents complexes CDK-cyclines ne sont pas élucidés pour l'ensemble des complexes, mais il semble que leur formation et leur activation, à des moments précis et ordonnés, permettent la progression dans le cycle et, notamment, le passage des différents points de contrôle du cycle. Ainsi la CDKI associée aux cyclines $A$ et $B$ est active au cours de la phase G2, alors que CDK2 associée à la cycline $\mathrm{A}$ est active au cours de la phase S. En ce qui concerne les CDK2 à CDK6, leur activité s'exprime au cours de la phase G1. CDK7, quant à elle, ne montre pas de spécificité de phase : associée à la cycline A elle forme le complexe CAK (cyclin activating kinase) actif pendant toutes les phases du cycle. CAK phosphoryle spécifiquement la thréonine conservée autour de la position 161 des CDK. On doit noter que vraisemblablement de nouvelles CDK seront encore découvertes puisque par exemple on ne connait pas les partenaires CDK des cyclines $\mathrm{C}, \mathrm{F}$ et $\mathrm{G}$. 


\section{L'activation des CDK : exemple de CDKI}

Une CDK isolée (non associée à une cycline) et non phosphorylée est inactive. Les sites de phosphorylation ont été déterminés pour cdc2 (CDKI), CDK2 et CDK4. La phosphorylation du résidu thréonine 161 (ou de son homologue dans les autres CDK) est essentielle pour une activité maximale du complexe CDK-cycline. En plus, il existe deux sites de phosphorylation "inhibitrice" sur les résidus thréonine en position 14 et tyrosine 15. Lors de la transition de la phase G2 à la phase $\mathrm{M}$, lors d'une mitose ou d'une méiose, la CDKI est pré-activée par la liaison de la cycline $B$ et la phosphorylation des 3 acides aminés, thréonine 161 et 14 , et tyrosine 15 . Ces phosphorylations font intervenir des kinases spécifiques : une kinase associée à la membrane nucléaire pour la thréonine 14, la kinase WEE1 pour la tyrosine 15 et le complexe CAK (CDK7) pour la T161. Lorsque la replication de l'ADN est terminée, et vérifiée, le passage de la phase G2 à la phase $M$ est rendu possible par activation d'une phosphatase CDC25C. Le complexe cycline B-CDKI est alors pleinement activé par élimination des groupes phosphates de la thréonine 14 et de la tyrosine 15 . Cette activation est amplifiée par l'activité kinase du complexe cycline B-CDKI amorçant un double rétrocontrôle positif : la suractivation de la phosphatase CDC25C et l'inhibition de la kinase WEE1.

\section{Que se passe-t-il en parallèle en ce} qui concerne l'unité régulatrice : la cycline, pendant cette transition G2/M ?

La cycline $\mathrm{B}$ commence à s'accumuler en début de phase G2 et forme des complexes préactifs avec CDKI. Au moment du passage en mitose, les complexes sont activés et induisent la phosphorylation de nombreux substrats tels les lamines nucléaires (ce qui conduit à la rupture de la membrane nucléaire) et les histones $\mathrm{H} 1$ et la topoisomérase II (ce qui permet la condensation et la décaténation de la chromatine). En fin de mitose, la formation de la membrane nucléaire des cellules filles nécessite l'inactivation des complexes cycline B-CDKI. Celle-ci résulterait de la dégradation de la cycline $B$. Cette dernière serait d'abord phosphorylée (par analogie avec ce qui se passe chez la levure). Cette phosphorylation favoriserait la fixation de molécules d'ubiquitine sur la cycline B. Le complexe serait alors dégradé dans des compartiments cellulaires spécialisés : les protéasomes.

On doit remarquer que ce schéma valable pour CDKI n'est sans doute pas parfaitement général. En effet, in vivo CDK2 est phosphorylée sur la tyrosine 15 en phase $S$, alors que l'activité kinase du complexe CDK2-cycline est maximale. Il semblerait donc que la régulation négative exercée par la phosphorylation sur la thréonine 14 et la tyrosine 15 ne soit pas aussi universelle que la régulation positive exercée par la phosphorylation sur la thréonine 161 .

Durant l'année 1993, il a été mis en évidence dans les cellules de mammifères une série de petites protéines capables de lier les complexes CDK-cyclines et d'inhiber leur activité kinase, les CDKI. En fait, le premier inhibiteur d'un complexe CDKcycline a été mis en évidence chez la levure $S$. Cerevisiae : c'est FAR1 pour «factor arrest" 1. La liaison de FAR1 au complexe cdc28-CLN (cdc28 est l'homologue de cdc2 et les CLN sont les cyclines G1 présentes chez la levure) entraîne l'inhibition de l'activité kinase du complexe cdc28-CLN. La liaison de FAR1 à cdc28-CLN est déclenchée par une phéromone, le facteur a qui active un récepteur membranaire couplé à une protéine $\mathrm{G}$ et mobilise une voie de signalisation analogue à celle de la MAP kinase dont nous allons reparler à propos de l'ovocyte. Outre le fait que ces protéines inhibitrices puissent participer à la régu- 
lation du cycle cellulaire en retardant l'activation des complexes CDK-cyclines spécifiques de la transition G1/S jusqu'au moment adéquat, leur dysfonctionnement pourrait être la cause de certains types de cancers.

\section{Quand intervient RB ?}

La transition entre les phases $\mathrm{G} 1$ et $\mathrm{S}$, qui fait intervenir les complexes cycline $\mathrm{E}$ CDK2 et cycline A-CDK2, est sous le contrôle des protéines codées par l'antioncogène rb (non fonctionnel dans les tumeurs de l'oeil de type rétinoblastome). $\mathrm{RB}$ est une famille de 3 protéines $\mathrm{p} 107$, p110 et p130. p110RB intervient au cours de la phase G1 : sous forme non phosphorylée elle neutralise l'action des facteurs de transcription E2F et DP1 qui contrôlent l'expression des protéines nécessaires au déroulement de la phase $\mathrm{S}$. Sa phosphorylation par le complexe cycline E-CDK2 libère ces facteurs de transcription, permettant ainsi la synthèse de l'ADN polymérase $a$, de C-MYC, de CDKI et d'enzymes impliquées dans la biosynthèse des nucléotides, comme la thymidylate kinase et la dihydrofolate réductase ; le passage en phase $\mathrm{S}$ est alors possible. Lorsque l'activité des facteurs E2F et DP1 n'est plus nécessaire, le complexe cycline A-CDK2 les inactive en réduisant leur affinité pour l'ADN par phosphorylation. Les protéines p107 et p130 neutralisent d'autres membres de la famille E2F qui agissent plus précocement au cours de la phase G1. Leur activité est régulée par les complexes cycline D-CDK. Les protéines $\mathrm{RB}$ pourraient également agir au cours de la phase G2 car leur taux de phosphorylation augmente à nouveau au cours de cette phase. A l'issue de la mitose les protéines $\mathrm{RB}$ sont recyclées par déphosphorylation.

\section{Quel est le rôle de la protéine p53 ?}

La protéine p53 joue un rôle essentiel dans le maintien de l'intégrité du génome. Son premier rôle est de bloquer le cycle cellulai- re lors de la détection d'une lésion de l'ADN. La phosphorylation de p53, par la kinase ADN-dépendante, permet sa stabilisation et l'augmentation de sa concentration nucléaire. Ce facteur de transcription modifie alors la capacité transcriptionnelle de la cellule : celle-ci est globalement réprimée, sauf pour les gènes qui possèdent dans leur région régulatrice un site de fixation spécifique pour p53. On observe alors l'expression des protéines p21, de la cycline $\mathrm{G}$ (une anti-cycline ?), puis finalement l'arrêt du cycle en phase G1.

Lorsqu'une lésion de l'ADN apparaît en phase G2, un épissage alternatif du gène p53 conduit à la synthèse de la protéine p53as qui semble posséder toutes les propriétés de p53 excepté celle de favoriser l'appariement de brins complémentaires d'ADN. L'arrêt du cycle cellulaire permettrait ainsi la réparation de l'ADN. p53 intervient en effet dans la réparation de l'ADN en interagissant avec les hélicases impliquées dans la réparation par excision, et en facilitant l'appariement de brins complémentaires d'ADN. Enfin, p53 peut favoriser le déclenchement de l'apoptose par induction de la protéine BAX.

\section{LE CONTROLE DE LA MEIOSE DANS L'OVOCYTE}

L'entrée en méiose de l'ovocyte se réalise durant la vie foetale et s'accompagne d'une synthèse d'ADN analogue à la phase $S$ d'une mitose. La plupart des ovocytes ont ensuite deux phases d'arrêt pendant la méiose : les caractéristiques varient selon l'espèce. Généralement la méiose se déroule jusqu'au stade diplotène de la première prophase et reste bloquée au stade diacinèse à l'intérieur de l'ovaire. Cette étape correspond à la phase G2 du cycle cellulaire. Elle est caractérisée par des chromosomes diffus, entourés par une membrane nucléaire que l'on appelle la vésicule germinale. La reprise de la méiose, qui s'opère à la puber- 
té, correspond à la transition G2/M et fait intervenir la dissolution de la membrane nucléaire (rupture de la vésicule germinale, GVBD pour germinal vesicle breakdown), la formation du fuseau de division et la ségrégation des chromosomes. L'ovocyte termine alors la première division de méiose en expulsant son premier globule polaire. Il poursuit ensuite sa maturation et s'arrête une seconde fois en métaphase de seconde division. La transition entre les deux métaphases de méiose n'est pas interrompue par une interphase. L'achèvement de la deuxième division de méiose est déclenché par la pénétration du spermatozoïde lors de la fécondation.

Ainsi, la première pause dans le processus de méiose est rompue par un signal hormonal qui arrive alors que l'ovocyte est encore dans l'ovaire, alors que la deuxième pause est rompue lors de la fécondation. Le rôle essentiel du MPF au début de la phase M a été mis en évidence lors d'études réalisées, pour la plupart, sur des ovocytes d'invertébrés et sur des cellules somatiques en mitose. Les connaissances sont moins nombreuses en ce qui concerne les mécanismes régulateurs de la méiose dans l'ovocyte du mammifère. Toutefois on peut tracer un schéma, sans doute encore imparfait, des connaissances de ces processus, qui peut différer, cependant, selon l'espèce à laquelle on s'adresse.

\section{Première pause au cours de la méio- se}

L'arrêt en G2/M s'effectue lorsque l'ovocyte contient de grandes quantités de p34cdc2 inactif, sous sa forme phosphorylée sur la tyrosine 15. Au contraire l'activité $\mathrm{MPF} / \mathrm{H} 1$ kinase $=\mathrm{p} 34 \mathrm{cdc} 2-$ cycline $\mathrm{B}=\mathrm{CDKI}$ - cycline $\dot{B}$, apparaît lors de la phase de rupture de la vésicule germinale, atteint un haut niveau lors de la métaphase I et disparaît de manière transitoire au moment de l'expulsion du premier globule polaire. Cette activité réapparaît à la métaphase II et reste élevée jusqu'à la fécondation. La nécessité d'une synthèse protéique à cette étape est fonction de l'espèce étudiée. Ainsi chez la palourde ou l'étoile de mer, l'application de l'hormone 1-méthyladénine entraîne la rupture de la vésicule germinale en 15 minutes. Dans l'ovocyte de grenouille, au contraire, bien que de grandes quantités de cycline $B 1$ et $B 2$ soient présentes, associées à la forme phosphorylée inactive de $\mathrm{p} 34 \mathrm{cdc} 2$, la rupture de la vésicule séminale n'a lieu que plusieurs heures après l'addition de l'hormone déclenchante qui est la progestérone. Or ce n'est pas une synthèse de cycline qui est requise. La protéine nécessaire pourrait être MOS. Pour ce qui est de l'ovocyte de souris, une synthèse protéique n'est pas nécessaire lors de la métaphase I, alors qu'elle l'est chez les porcins, les bovins et les ovins. La reprise de la méiose de l'ovocyte de mammifère également nécessite la déphosphorylation de la tyrosine 15 de p34cdc2. En effet, les expériences utilisant le vanadate, qui est un inhibiteur des tyrosine phosphatases, bloque la reprise de la méiose dans l'ovocyte de rat et de souris. MOS ne semble pas nécessaire chez la souris pour la reprise de la méiose. La raison pour laquelle les ovocytes sont bloqués en G2/M n'est pas connue avec certitude. Pour ce qui est de l'ovocyte d'étoile de mer ou de palourde, il semblerait que le processus d'activation de cdc25 ne soit pas fonctionnel, ce qui empêcherait la déphosphorylation de p34cdc2 sur la tyrosine 15 bloquant ainsi l'activité MPF.

\section{Arrêt au cours de la métaphase II}

L'ovocyte de grenouille, et de même celui des mammiferes, s'arrête une seconde fois à la métaphase de seconde division. A ce moment pourtant, l'activité MPF est élevée et la dégradation des cyclines est inhibée. Ce second arrêt est dû à l'activité CSF (pour "cytostatic factor"). Or la protéine MOS a le même effet que le CSF car elle est capable d'arrêter la mitose à la métaphase, 
alors qu'il y a une activité p34cdc2 importante. Actuellement, il semble raisonnable de penser que CSF est en partie ou en totalité MOS et que le second arrêt en métaphase est dû à la transcription de mos quand l'ovocyte mature. Cependant MOS est aussi nécessaire à la maturation de l'ovocyte de grenouille. En effet il est exprimé à ce stade du développement de l'ovocyte, et cette première pause en prophase I ne peut être rompue en présence d'oligonucléotides antisens pour mos. Ceci suggère donc que dans cette espèce (contrairement à la souris), MOS est nécessaire à la transition G2/M. MOS agit probablement en activant la MAP kinase. La MAP kinase elle-même a une activité CSF.

Les membres de la famille des MAP kinases (pour "mitogen activated proteins") sont des sérine/thréonine kinases qui sont activées par phosphorylation sur des résidus tyrosine et thréonine. Leurs poids moléculaires sont autour de 42-44 kDa. Des résultats récents ont montré que la MAP kinase est activée au cours de la maturation de l'ovocyte et qu'elle régule la méiose. L'activation de la MAP kinase précède l'activation de $\mathrm{p} 34 \mathrm{cdc} 2$ lors de la première méiose. Le blocage de l'activité MAP kinase bloque également la rupture de la vésicule germinale. Chez la souris, contrairement à l'étoile de mer, l'activité MAP kinase reste élevée pendant toute la période de maturation de l'ovocyte et durant l'arrêt en métaphase II. Cette activité chute après la fécondation et pendant la première mitose lors de la formation du pronucleus.

MOS stimule l'activité MAP kinase dans l'ovocyte de grenouille mais n'active pas p34cdc2. Le couple MOS/MAP kinase pourrait avoir deux effets : produire les caractéristiques du deuxième cycle de méiose, c'est-à-dire celui où la phase $\mathrm{S}$ est exprimée, et être la cause du blocage en métaphase II. La cascade de phosphorylations qui relient MOS à la MAP kinase dans l'ovocyte n'est que partiellement élucidée.
Dans les cellules somatiques, la MAP kinase est activée par une MAP kinase kinase qui est activée indirectement par les Raf et Ras kinases. Or dans l'ovocyte de grenouille, MOS agit par l'intermédiaire d'une MAP kinase kinase. Cependant, chez la souris, bien que Raf1 soit présent dans l'ovocyte immature, il n'est pas phosphorylé (activé) au moment de l'activation de la MAP kinase.

\section{Quels sont les facteurs de signalisa- tion cellulaire impliqués dans ces régulations?}

\section{a) Première pause de méiose}

Chez l'étoile de mer, la 1-méthyl adénine induit la maturation, probablement via une chute de la teneur de l'ovocyte en AMPc. Pour ce qui est de l'ovocyte de mammifêre, la maturation méiotique se réalise spontanément lorsque l'ovocyte est extrait du follicule ; il n'est pas besoin d'un stimulus hormonal pour réinitier la méiose et atteindre la métaphase II. Par contre, la maturation spontanée, qui se produit in vitro, est stoppée si on ajoute au milieu de culture un analogue de l'AMPc ou un inhibiteur des phosphodiestérases. Il semble donc que, là encore, l'AMPc soit impliqué dans le blocage de l'ovocyte en prophase I. D'ailleurs, on observe une chute significative de la teneur de l'ovocyte en AMPc juste avant la rupture de la vésicule germinale. Cependant il faut remarquer que les esters de phorbol ou le diacylglycérol, qui activent la PKC, inhibent également la maturation ovocytaire spontanée qui se réalise in vitro, mais ceci sans modification de la concentration ovocytaire en AMPc.

Le Vérapamil et la tétracaïne, deux inhibiteurs du transport transmembranaire du $\mathrm{Ca}++$, empêchent transitoirement la rupture de la vésicule germinale in vitro. $\mathrm{De}$ plus, l'augmentation des concentrations extracellulaires de $\mathrm{Ca}++$ diminue l'efficacité de l'AMPc à maintenir l'ovocyte bloqué. Il apparaît donc y avoir un effet antagoniste entre le Ca++ et l'AMPc à ce niveau. 


\section{b) Arrêt en métaphase II (2ème pause)}

Chez les mollusques et les ascidiens, dont les ovocytes s'arrêtent en métaphase I, comme chez les amphibiens et les mammifères, dont l'ovocyte est bloqué en métaphase II, c'est la fécondation qui déclenche une augmentation du Ca++ qui est le signal de reprise de la méiose. Ce mécanisme est le mieux compris chez la grenouille. L'hypothèse est la suivante : le Ca++ active la calmoduline, qui elle-même active la calmoduline kinase II, qui active à son tour la destruction de la cycline et la dégradation de MOS. En effet les inhibiteurs de la calmoduline et de la Cam kinase II empêchent l'effet du Ca+t. Inversement, la microinjection d'une forme constitutionnellement active de la Cam kinase II lève l'arrêt de la méiose en absence de toute augmentation de $[\mathrm{Ca}++]$. On doit cependant remarquer qu'il n'a pas été établi de relation directe entre la Cam kinase II et la destruction de la cycline. D'autre part, la quantité de cycline diminue avant la protéine MOS et donc la levée d'inhibition n'est pas symétrique du mécanisme qui établit cet arrêt dans la méiose. Enfin on ne sait pas très bien comment le spermatozoïde induit l'augmentation du $[\mathrm{Ca}++]$.

\section{c) Conclusions}

Il semble désormais bien établi que l'arrêt en méiose peut être expliqué en terme d'activation et d'inactivation des protéines qui contrôlent le cycle cellulaire, et en particulier p34cdc2, MOS et MAP kinase. Le lien entre p34cdc2 et la MAP kinase n'est pas bien compris ; leurs activités respectives augmentent en parallèle au cours de la première division de méiose, mais dans les ovocytes qui ont un second arrêt lors de la méiose, l'activité de p34cdc2 diminue, tandis que l'activité MAP kinase reste élevée. Les ovocytes requièrent l'activation de p34cdc2 pour atteindre la métaphase I et l'inactivation de p34cdc2 pour passer l'anaphase du cycle cellulaire suivant. Ils nécessitent l'activation de MOS/MAP kinase pour rompre la première pause de la méiose (mais probablement pas dans l'ovocyte de souris) et encore pour induire la pause en métaphase II. Enfin, les relations entre MOS/MAP kinase et l'activation de p34cdc2 à l'échelle moléculaire sont très mal connues.

\section{LE CONTROLE DE LA MEIOSE DANS LA LIGNEE GERMINALE MALE}

De même que pour la gonade femelle, les cellules génitales primordiales colonisent les crêtes germinales au cours de l'embryogenèse. Une fois dans la gonade, les cellules germinales mâles prolifêrent alors que les cordons séminifères qui les entourent se développent. Au cours du développement foetal, elles grossissent mais cessent de se multiplier. Les mitoses des gonocytes ne reprennent que quelques jours après la naissance, puis ceux-ci se différencient en spermatogonies.

\section{Quels sont les gènes impliqués dans le déroulement de la méiose?}

Un grand nombre de gènes exprimés uniquement dans les cellules germinales mâles, ou dont le type d'expression est particulier à ces cellules, ont été identifiés. Cependant peu d'études ont été réalisées en ce qui concerne les gènes potentiellement impliqués dans le déroulement des mitoses des cellules germinales mâles ou de la méiose.

\section{C-mos}

Dans le testicule de la souris, c-mos est principalement exprimé sous la forme d'un transcrit de $1,7 \mathrm{~kb}$, spécifique du testicule dans les spermatides jeunes. Au contraire les anticorps reconnaissant la protéine MOS de $43 \mathrm{kD}$ montrent que celle-ci est abondante dans les spermatocytes pachytènes et peu ou pas présente dans les cellules post-méiotiques. Ces résultats indiquent donc une régulation, non seulement 
au niveau transcriptionnel mais aussi traductionnel de MOS. On doit également remarquer que MOS est également exprimé par les cellules de Leydig et de Sertoli. Au contraire de ce qui est connu du rôle de MOS dans l'ovocyte, il n'existe pas de données sur le rôle de MOS lors de la spermatogenèse, bien que son abondance dans les pachytènes suggère un rôle dans le processus de la méiose. Cependant des résultats très récents font état que l'expression de $\mathrm{v}$ mos sous le contrôle du promoteur PGK2 dans des souris transgéniques, entraîne un arrêt de la spermatogenèse en métaphase I. Par contre, la surexpression de c-mos ne cause pas de stérilité.

\section{Gènes des cyclines}

\section{a) Cycline B}

Chez les mammiferres, les cyclines B1 et B2 sont exprimées lors de la transition G2/M. Ces deux cyclines sont également exprimées dans le testicule et sont spécifiques des cellules germinales. Par hybridation in situ, il apparaît que l'ARN messager de la cycline B1 est surtout présent dans les spermatides rondes, ce qui semble surprenant puisque ces cellules ont terminé leur méiose. Toutefois les quantités de protéine cycline B1 et son activité kinasique sont maximales dans les spermatocytes pachytènes. Ceci suggère pour la cycline B1 comme pour MOS, une régulation post-transcriptionnelle importante, et que les ARN messagers présents dans les spermatides rondes pourraient ne pas être traduits.

Pour ce qui est des transcrits codant la cycline B2, leur niveau apparait maximum dans les pachytènes âgés, c'est-à-dire juste avant la première division de méiose.

\section{b) Cycline A}

Le rôle de la cycline A, plutôt au cours de la mitose ou au cours de la méiose, n'est pas complètement éclairci. Il apparaît y avoir au moins deux gènes de cycline $\mathrm{A}$. Le gène CYC A1 est très fortement exprimé dans le testicule adulte et seulement dans les cellules en méiose ou juste avant. Au contraire, le gène CYC A2 n'est détecté que dans les cellules en mitose (spermatogonies) et les spermatocytes préleptotènes, c'est-àdire au moment de la synthèse d'ADN de la méiose. Ainsi, le gène de la CYC A1 apparaît plutôt impliqué dans le contrôle de la phase $\mathrm{M}$ et celui de la CYC A2 plutôt dans le contrôle de la phase S.

\section{c) Cycline D}

Les gènes codant pour les cyclines D1, D2 et D3 sont exprimés dans le testicule, de même que dans l'ovaire, mais leurs profils d'expression sont très différents dans ces deux glandes. La cycline D1 est fortement exprimée dans le testicule au début de la vie post-natale et chez les animaux adultes dépourvus de cellules germinales. Les études en hybridation in situ confirment ces résultats en ne détectant une expression de l'ARN messager correspondant que dans les cellules de Sertoli.

Le gène codant la cycline D2, quant à lui, apparaît exprimé de manière faible dans tous les types cellulaires du testicule. La cycline D3, comme les autres membres de cette famille, est particulièrement active pendant la phase G1. Dans le testicule, l'expression du gène CYC D3 apparaît maximale dans les spermatides rondes. Ce résultat est quelque peu surprenant dans la mesure où les spermatides rondes ne sont pas des cellules qui se divisent. Mais là encore, seule l'expression du gène a été étudiée et l'hypothèse d'un décalage entre l'expression des ARNm et de la protéine ne peut être écartée.

\section{d) Autres types de cyclines}

Très peu de données ont été obtenues jusqu'à présent concernant l'expression des autres types de cyclines dans le testicule.

\section{Kinases dépendant des cyclines}

Peu d'études ont été réalisées pour tenter d'élucider le rôle des CDKs dans la lignée 
germinale mâle. Il faut, à ce propos, rappeler que les mécanismes régulateurs de leur activité sont essentiellement des mécanismes post-traductionnels puisqu'ils caractérisent des réactions de phosphorylation et déphosphorylation, ainsi que leur association avec des cyclines spécifiques, et nécessitent donc des méthodes autres que la biologie moléculaire pour être étudiés. En outre, des observations récentes suggèrent que les CDKs pourraient être impliquées dans des phénomènes autres que la régulation du cycle cellulaire.

Les gènes codant pour CDKI, CDK2, CDK3, CDK4 et CDK5 sont exprimés dans le testicule. L'expression de ces gènes apparaît dépendante du type cellulaire et du stade de développement, mais en outre, cette expression ne semble pas strictement reliée à une activité de prolifération. Ainsi CDKI et CDK2 sont fortement exprimées dans les spermatocytes pachytènes et diplotènes, mais les gènes codant pour les CDKI à 5 sont également exprimés dans les cellules de Sertoli adultes alors que celles-ci ne se multiplient plus.

\section{Protéines phosphatases cdc25}

Chez les mammiferes trois homologues du gène cdc25 ont été identifiés : cdc25A, cdc25B et cdc25C. Les ARNm de cdc25A apparaissent au début de la phase G1 et sont à leur niveau maximum en milieu et en fin de phase G1. La protéine correspondante est active lors de la transition G1-S et pourrait également être importante en fin de phase M. Les ARNm de cdc25C sont en quantité importante en phase G2 et la protéine active à la transition G2-M. Le stade du cycle cellulaire où agirait cdc25B n'est pas connu. Dans le testicule de la souris adulte, l'expression de cdc25C apparait restreinte aux cellules germinales et en particulier aux spermatocytes pachytènes et diplotènes, ce qui apparaît tout à fait compatible avec un rôle lors de la transition G2-M. Ces transcrits persistent cependant dans les spermatides rondes et allongées. cdc25B, au contraire, apparaît fortement exprimé dans les cellules somatiques dont la plupart, dans le testicule adulte, ne sont pas en phase de prolifération. L'expression de cdc25A dans le testicule n'a pas encore été publiée à notre connaissance.

\section{Quels sont les hormones et les fac- teurs locaux potentiellement impli- qués dans la régulation de la méiose chez le mâle?}

Les données de la littérature concernant la régulation de la méiose par les hormones et/ou les facteurs de croissance sont extrêmement réduites. De nombreuses études ont eu pour objectif de déterminer la nature des facteurs de croissance et des cytokines présents dans le testicule, la localisation cellulaire de leur expression et de celle de leurs récepteurs à l'intérieur du tube séminifêre. Cependant la démonstration directe d'un effet de telle ou telle substance sur les événements moléculaires impliqués dans le déroulement de la méiose sont rares pour une raison essentielle : la quasi absence de systèmes in vitro permettant d'expérimenter. A notre connaissance, seule l'école finlandaise, animée par Parvinen, a pu obtenir quelques résultats dans ce domaine, grâce à un système de culture de segments de tubules de stades définis de l'épithélium séminifère. Ainsi ce groupe a-t-il pu montrer que la FSH, l'interleukine-1a et le MIP-1a (macrophage inflammatory protein) stimulent l'incorporation de thymidine tritiée dans les segments de stades VII à IX par les spermatocytes en stade préleptotène. Inversement, l'interleukine- 6 apparaît exercer, dans ce système, une action inhibitrice sur la synthèse d'ADN méiotique. On doit cependant noter que les effets observés sont souvent de faible amplitude, ceci résultant peut-être, en partie, d'une faible accessibilité du facteur ajouté aux cellules cibles, du fait même de leur organisation à l'intérieur du segment de tube cultivé. 
Il a enfin été postulé que les cellules de Sertoli sont la source d'une substance inhibant l'entrée en méiose des cellules germinales (MIS). Cependant, ni l'identité du MIS, ni son mécanisme d'action ne sont actuellement connus.

\section{Essais de mise au point de cocul- tures cellules somatiques-cellules ger- minales testiculaires}

Un des objectifs de notre laboratoire, au cours des dernières années, a été de mettre en place deux systèmes de coculture permettant à certaines étapes de la différenciation des cellules germinales mâles de s'effectuer in vitro. Le rat et la souris sont, actuellement, nos modèles animaux expérimentaux. Chez ces espèces, en effet, les différentes étapes de la spermatogenèse ont été très bien définies. De plus, l'apparition des différents types de cellules germinales, au moment de la puberté, se fait de manière séquentielle et avec une cinétique relativement précise. C'est aussi chez ces espèces que les techniques de séparation des différents types de cellules germinales sont le mieux établies (élutriation, gradients de ficoll, etc.). Enfin, la plupart des gènes dont on sait qu'ils s'expriment de façon séquentielle ou spécifique lors de la différenciation des cellules germinales mâles ont été clonés chez la souris ou chez le rat. Nous avons récemment validé deux méthodes de coculture cellules somatiques des tubes séminifèrescellules germinales. La première consiste à cocultiver des spermatocytes pachytènes ou des spermatides rondes préparées par élutriation sur un tapis de cellules de Sertoli en chambres bicamérales. Dans la seconde méthode nous mettons en culture des petits fragments de tubules séminifères ; au bout de quelques jours, les cellules intratubulaires migrent et colonisent la totalité de la plaque de culture. Ainsi on conserve beaucoup mieux in vitro les communications intercellulaires présentes in vivo et la totalité des types de cellules germinales présentes dans le tubule au moment de son ensemencement peut être étudiée. Le premier type de culture permet de maintenir une viabilité des cellules supérieure à $60 \%$ pendant 2 semaines. Le second conserve une viabilité des cellules voisine de 70 à $80 \%$, pendant 3 semaines. L'évolution des cellules préméiotiques (spermatocytes) a été étudiée in vivo, au cours de la période de mise en place de la spermatogenèse et dans ces deux types de coculture en étudiant :

- l'expression de gènes spécifiques des cellules germinales pré- et post-méiotiques (p19 et TH2B sont fortement exprimés avant la méiose et s'éteignent après, alors que TP1 et TP2, peu ou pas exprimés dans les spermatocytes, sont fortement exprimés après la méiose dans les spermatides rondes),

- la ploïdie des populations cellulaires,

- l'histologie et l'immunocytologie des cultures après marquage in vivo à la 5bromo-2'-déoxyuridine (BrdU).

Les deux types de cocultures permettent de conserver une activité transcriptionnelle des spermatocytes pendant 2 à 3 semaines. Ils permettent également de réaliser une grande partie de la méiose in vitro puisque les spermatocytes pachytènes des stades $\mathrm{V}$ à VIII dans le premier modèle de coculture, et des stades IV à VI dans le second modèle, évoluent en spermatides in vitro. A terme, l'utilisation combinée de ces deux systèmes in vitro devrait permettre d'étudier un certain nombre des modifications post-traductionnelles qui affectent les protéines régulatrices du cycle cellulaire lors de la mitose ou de la méiose. Ces deux systèmes seront également utiles pour aborder de manière simplifiée (par ajout ou blocage, de leur expression ou de leur action) l'incidence de facteurs locaux sur le déroulement de certaines étapes de la spermatogenèse. 


\section{CONCLUSIONS}

Les études réalisées au cours des dix dernières années ont montré que la mitose et la méiose partagent un grand nombre de mécanismes moléculaires régulant les différentes étapes de ces processus. Ces données ont été obtenues d'abord sur des modèles expérimentaux tels que la levure ou la drosophile, avant d'être étendues aux eucaryotes supérieurs. Pour ce qui est de la méiose, l'ovocyte de mollusque ou d'amphibien s'est avéré être un modèle de choix du fait de la grosseur de cette cellule et de la possibilité de l'obtenir en grande quantité. Les résultats concernant la lignée germinale mâle sont infiniment plus rares. En effet, ces recherches souffrent, en particulier, de la difficulté d'obtenir des cellules germinales d'un stade précis de leur différenciation en grande quantité et d'un manque de systèmes in vitro permettant d'appréhender l'importance des régulations post-traductionnelles impliquées dans ces processus, et d'identifier les facteurs hormonaux ou locaux les induisant. Il est donc essentiel de relever ces deux défis rapidement pour une meilleure connaissance d'un phénomène biologique fondamental pour la survie des espèces.

\section{REFERENCES}

Sur les gènes régulateurs du cycle cellulaire

1. CAROSELLA E.D., LETEURTRE F. : La danse des kinases. Biofutur, octobre 1995 : 29-34.

2. DARBON J.M., FESQUET D., CAVADORE J.C. : De nouveaux régulateurs du cycle cellulaire : les protéines modulatrices des complexes CDKcyclines. Médecine/Sciences, $1995 ; 11: 349$ 356.

Sur le contrôle de la méiose dans l'ovocyte

3. DEKEL N. : Molecular control of meiosis. Trends in Endocrinology and Metabolism, $1995 ; 6: 165$ 169.

4. WHITAKER M. : Control of meiotic arrest. Reviews of Reproduction, 1996, $1:$ 127-135.
Sur le contrôle de la mitose et de la méiose dans la lignée germinale mâle

5. WOLGEMUTH D.J., REE K., WU S., RAVAIK S.E : Genetic control of mitosis, meiosis and cellular differentiation during mammalian spermatogenesis. Reprod. Fertil. Dev., 1995 ; 7 : 669-683.

6. PARVINEN M., SÖDER O., MALI P., FRÖYSA B., RITZEN E.M. : In vitro stimulation of stage specific DNA synthesis in rat seminiferous tubule segments by interleukin-1a. Endocrinology, $1991 ; 129: 1614$ 1620.

7. HAKOVIRTA H., VIERLA M., WOLPE S.D., PARVINEN M. : MIP-1a is a regulator of mitotic and meiotic DNA synthesis during spermatogenesis. Mol. Cell. Endocrinol., 1994 ; 99 : 119-124.

8. HAKOVIRTA H., SYED V., JEGOU B., PARVINEN M. : Function of interleukin-6 as an inhibitor of meiotic DNA synthesis in the rat seminiferous epithelium. Mol. Cell. Endocrinol., 1995 ; 108 : 193 198.

9. HENRIKSEN K., KANGASNIEMI M., PARVINEN M., KAIPIA A., HAKOVIRTA H. : In vitro, FSH prevents apoptosis and stimulates DNA synthesis in the rat seminiferous epithelium in a stage-specific fashion. Endocrinology, $1996 ; 137: 2141-2149$.

\section{ABSTRACT \\ Mechanisms of control of meiosis in male germ cells \\ P. Durand}

In 1883 , it was discovered that whereas the fertilized egg of a particular worm (Parascaris Equorum) contains four chromosomes, the nucleus at the egg and that of the sperm each contains only two chromosomes. This finding implied that germ cells must be formed by a special kind of nuclear division in which the chromosome complement is precisely halved. This type of division is called meiosis. Meiosis and mitosis share many regulatory elements. Among them, are the cyclindependent kinases (CDK) and the cyclins which control all transitions of the cell cycle. The activity of the CDKs is regulated by their association to specific cyclines and by phosphoryla- 
tion/dephosphorylation reactions. Moreover, recent reports reveal the existence of a variety of small proteins which bind to and modulate G1/S CDKcyclin complexes : the CKI. Two more proteins appear of great importance in regulating the cell cycle : $\mathrm{Rb}$ and $\mathbf{p 5 3}$. The central role of MPF (maturation producing factor $=$ M-phase promoting factor) has been established, mostly by studies conducted on either invertebrates oocytes and eggs that resume the meiotic division. MPF is the cyclin B-p34cdc2 kinase. As for the cytostatic factor (CSF), it is reasonably certain that it is, in part or entirely, MOS. The cell cycle signalling mechanisms in oocytes include cAMP, $\mathrm{Ca}^{++}$and the agonists of PKC. Much less informations on the control of meiosis in spermatogenesis are available. Indeed, it is difficult to get rather high number of germ cells at some precise steps of their maturation and there is a lack of culture system allowing in vitro germ cell differentiation. However, most of the genes involved in the regulation of the cell cycle are expressed in the testis (c-mos, cyclins, CDK, cdc25 ...). Their expression has striking cellular, lineage and developmental specificity. It has also been shown that FSH, interleukin-1a, and MIP-1a enhance stagespecific DNA synthesis in rat seminiferous tubule segments, while interleukin-6 decreases it. In our laboratory, we have settled recently two cell culture systems allowing the expression of germ cell specific genes for 2 to 3 weeks. This should help to study more easily the genetic control of meiosis during spermatogenesis and to understand better which growth factors and/or cytokines are really important for the regulation of this process.

Key-words : meiosis, cell cycle, oocyte, spermatogenesis. 